\title{
ALGUMAS REFLEXÕES SOBRE O EXERCÍCIO DA PESQUISA NA EDUCAÇÃO FÍSICA
}

\author{
Priscilla Maia da Silva \\ Universidade Estadual de Londrina, Londrina, Paraná, Brasil \\ Keila Cristina de Moraes Lima Kubo \\ Universidade Estadual de Londrina, Londrina, Paraná, Brasil \\ Thiago Viana Camata \\ Universidade Estadual de Londrina, Londrina, Paraná, Brasil \\ Jeane Barcelos Soriano \\ Universidade Estadual de Londrina, Londrina, Paraná, Brasil
}

\begin{abstract}
Resumo
O objetivo foi realizar algumas reflexões sobre o que tem sido discutido pela comunidade científica em geral, e da área da Educação Física, sobre as práticas questionáveis (PQs) empregadas no exercício da pesquisa científica. Dentre as inúmeras estratégias para levar vantagem no sistema que avalia a produtividade em pesquisa, as PQs aparecem com pouca frequência como principal objeto de investigação em pesquisas na área da Educação Física. A abordagem qualitativa e tipo de pesquisa bibliográfica caracterizam a metodologia utilizada. A análise de conteúdo foi empregada para análise dos documentos selecionados. Consideramos que é preciso ampliar os estudos sobre a temática, visando elaborar meios efetivos para prevenção de práticas questionáveis na pesquisa.

Palavras-chave: Má Conduta Científica. Indicadores de Produção Científica. Educação Física e Treinamento.
\end{abstract}

\section{Introdução}

\begin{abstract}
A cada ano o Brasil vem conquistando destaque no cenário científico mundial com seus índices de produtividade científica. Nas duas bases de dados de reconhecimento internacional adotadas como referência pelo governo brasileiro para acompanhar o avanço da ciência nacional, o país tem obtido bons resultados. Em 2009, o país publicou 32.100 artigos em periódicos indexados no Institute for Scientific Information (ISI) (BRASIL, 2012). Na base Scopus, o país foi o $15^{\circ}$ mais produtivo do mundo em 2010, com 45.189 artigos (SCOPUS, 2010). Não há dúvidas que muito desse crescimento está diretamente relacionado com o desenho e as estratégias de implementação de políticas científicas voltadas para a quantificação do conhecimento.
\end{abstract}


Como um dos possíveis desdobramentos tem-se o aumento de certas práticas, que a literatura especializada tem chamado de "questionáveis" (COMMITTEE..., 2009; PANEL..., 1992; STENECK, 2006; JOHN; LOEWENSTEIN; PRELEC, 2011). As práticas questionáveis (PQs) mais conhecidas são o plágio, a falsificação e fabricação de dados e/ou resultados (STENECK, 2006; DE VRIES; ANDERSON; MARTINSON, 2006; MACRINA, 2005; ROCHA; BENEDETTI, 2009). Outras PQs também têm sido citadas, a saber: (a) autoplágio (CNPq, 2011; BENOS et al., 2005; CARNEIRO; CANGUSSÚ; FERNANDES, 2007); (b) fragmentação de um mesmo estudo em vários artigos (CNPq, 2012a; BENNETT; TAYLOR, 2003; BOSSI, 2010; CASTIEL; SANZ-VALERO, 2007; KUMAR, 2008; MABE; AMIN, 2002; MOJON-AZZI; MOJON, 2004; RIGHETTI, 2010; ROCHA; BENEDETTI, 2009) e; (c) autoria presenteada (BENNETT; TAYLOR, 2003; CASTIEL; SANZ-VALERO, 2007; MONTENEGRO, 1999; BUSSATO-FILHO, 2002; GRIEGER, 2005; ROCHA; BENEDETTI, 2009). Também, tem sido colocada como PQ usada para aumentar as chances de aceitação de um trabalho em um periódico, a citação de artigos do mesmo periódico, bem como incluir na autoria pessoas experientes em uma determinada área apenas para facilitar a aprovação do artigo (KEMPERS, 2002; BENNETT; TAYLOR, 2003; FEESER; SIMON, 2008; LOVISOLO, 2007; MONTEIRO et al., 2004).

Essas são algumas estratégias que podem estar sendo utilizadas por pesquisadores "para aumentar artificialmente o número de publicações, bem como de contagem de citações para aumentar o 'impacto' da publicação" (KUMAR, 2008, p.215, tradução nossa). Com isso, as PQs têm sido chamadas de "esteróides" do mundo acadêmico por ser um modo postiço de melhorar o desempenho científico (JOHN; LOEWENSTEIN; PRELEC, 2011). Assim, compreendemos como PQs as ações que vão contra os princípios da ética em pesquisa, como não realizar um trabalho genuíno ou não colaborar efetivamente em um estudo, o que caracteriza uma forma artificial de inflar os índices de produtividade em pesquisa.

É possível que alguns pesquisadores estejam usando um extenso leque de PQs e talvez um dos principais motivos possa ser a conquista de reconhecimento e prestígio no cenário acadêmico e científico (BOURDIEU, 1976). Por sua vez, como decorrência a busca por financiamento e vantagens na carreira são aspectos que, também, po- 
dem influenciar os indivíduos a utilizarem PQs (ANGELL,1986; CNPq, 2011; KEMPERS, 2002).

Alguns países contam com instituições (criadas nas décadas de 1980 e 1990) para identificar e julgar casos de PQs que acontecem no exercício da pesquisa científica. O Office of Research Integrity - ORI (Estados Unidos) foi criado em 1989; o Research Ethics Council (Finlândia) em 1991; o Committee on Scientific Dishonesty (Dinamarca) em 1992; o National Committee for the Evaluation of Dishonesty in Health Research (Noruega) em 1994; e a Délegation à la I'Intégrité Scientifique (França) em 1999 (FARTHING, 2004).

No Brasil, apenas em 2012 o Conselho Nacional de Desenvolvimento Científico e Tecnológico (CNPq) criou a Comissão Permanente de Integridade Científica, responsável por duas linhas de ação. A primeira engloba a adoção e coordenação de ações preventivas e educativas, que abrange: (a) inclusão de disciplinas sobre o tema em cursos de graduação e pós-graduação e; (b) divulgação de material educativo sobre os diferentes tipos de má-conduta em pesquisa. A segunda é destinada a analisar casos em que existam questionamentos quanto à integridade da pesquisa produzida e disseminada por pesquisadores financiados pelo $\mathrm{CNPq}$, propondo medidas punitivas quando for o caso (CNPq, 2011; 2012a; 2012b).

A percepção de que esse problema existe e precisa ser abordado parece estar crescendo entre a comunidade científica brasileira. Além do CNPq, a Fundação de Amparo à Pesquisa de São Paulo (FAPESP) já havia publicado em setembro de 2011, o Código de Boas Práticas Científicas para servir de guia de conduta para aqueles pesquisadores que são beneficiários de financiamento desta agência (CASTRO, 2011).

Apesar do desenvolvimento científico, em termos do volume de publicações, não existem informações no Brasil relativas ao número de casos e nem dados consolidados sobre os tipos de PQs que podem estar sendo adotadas no processo de produção científica, principalmente na área da Educação Física (EF). Um estudo publicado em 2010 mostrou que, de 183 disciplinas ofertadas nos Programas de PósGraduação (PPGs) da Grande Área das Ciências da Saúde, no qual a EF está inserida, 80,4\% eram omissas ao tema da integridade científica. Apenas os PPGs da Medicina, Odontologia e Enfermagem demonstraram maior preocupação com o assunto da ética em pesquisa científica, incluindo temas sobre conduta inadequada em pesquisa 
(SANTANA, 2010). Todavia, o tema ainda gera pouco interesse como objeto de investigação original, o que dificulta a elaboração de um quadro analítico e crítico daquilo que vem sendo produzido na área da EF (KUBO et al., 2011).

É possível que a preocupação dos pesquisadores

[...] esteja longe da visão hagiográfica da ciência que é desmentida por tudo o que se conhece da verdade da pesquisa: os plágios, o roubo de idéias, as querelas de prioridades e tantas outras práticas que são tão antigas quanto a própria ciência (BOURDIEU, 2004, p.31).

Adotamos como hipótese que, no meio científico, alguns pesquisadores podem estar fazendo o uso de inúmeras PQs para conquistar ou manter seu prestígio no campo científico e, com isso, obter vantagens nas regras da produtividade em pesquisa. Por decorrência, faz-se necessário uma reflexão sobre as implicações das PQs no sistema de concessão de auxílio à pesquisa e na comunicação científica. Com isso, o objetivo foi realizar algumas reflexões sobre o que tem sido discutido pela comunidade científica, em geral, e da área da EF, sobre as PQs empregadas no exercício da pesquisa científica.

\section{Encaminhamento metodológico}

A abordagem qualitativa norteou a condução do estudo, uma vez que a interpretação não está orientada para o controle de variáveis, que necessitam de valores (MINAYO, 1994; BAUER; GASKELL; ALLUN, 2002; RICHARDSON, 1985; MARCONI; LAKATOS, 2003; TURATO, 2005). Como o propósito foi colocar o leitor em contato com as discussões já realizadas sobre um determinado assunto, este estudo caracterizou-se como uma pesquisa bibliográfica, por ser o estágio inicial de apresentação do que tem sido publicado sobre o tema investigado (MARCONI; LAKATOS, 2003; MOREIRA, 2004; SÁ-SILVA; ALMEIDA; GUINDANI, 2009). Com isso, a pesquisa pode ser considerada uma revisão opinativa, uma vez que é um tipo de revisão no qual "os autores resumem, analisam e sintetizam as informações disponibilizadas na literatura, mas não seguem necessariamente uma metodologia pré-definida" (MANCINI; SAMPAIO, 2006, p.0). 
Artigos científicos, livros e artigos de opinião publicados em revistas científicas e jornais foram os tipos de documentos selecionados para o estudo. A coleta de dados aconteceu durante o ano de 2011. A busca de documentos foi realizada em livros sobre o tema, em bases de dados on-line de periódicos científicos (Portal de Periódicos da Capes, Web of Science e Scielo), bem como em bases de artigos científicos (Google Acadêmico e Mendeley). Pela característica da pesquisa, as referências dos artigos inicialmente encontrados também serviram de fonte de coleta de dados (LUNA, 1999; SÁ-SILVA; ALMEIDA; GUINDANI, 2009). Os documentos foram escolhidos por contribuir com informação pertinente para o tema pesquisado. A análise de conteúdo foi empregada como método de análise dos dados (BARDIN, 1977). As categorias de análise temática foram organizadas a posteriori, tendo como núcleo de sentido as práticas questionáveis em pesquisa.

\section{Práticas questionáveis: quais são e como são definidas}

O CNPq (2011) reconheceu como modalidades de fraude ou má conduta apenas a fabricação, falsificação, plágio e autoplágio. No cenário internacional, a fabricação, falsificação e plágio são conhecidas como FFP e são as mais discutidas em estudos sobre conduta inadequada (ANDERSON, 2008; DE VRIES; ANDERSON; MARTINSON, 2006; FANELLI, 2009; KUMAR, 2008; MARTINSON; ANDERSON; DE VRIES, 2005; ORI, 2008; STENECK, 2002; 2006).

Além da FFP, os estudos brasileiros também têm citado outras PQs, como os problemas de autoria, duplicação de artigo, manipulação de dados e citação (BUSSATO-FILHO, 2002; AZEVÊDO, 2006; CARVALHO, 2009; GRIEGER, 2007; JOB; MATTOS; TRINDADE, 2009; PETRONIAU, 2002; 2010; RODRIGUES; CRESPO; MIRANDA, 2006; TAVARES-NETO, 2009; VASCONCELOS et al., 2009). Diante da diversidade de estratégias empregadas para elevar a produtividade em pesquisa, como saber qual ação é uma conduta duvidosa? A seguir serão descritas as principais $\mathrm{PQs}$ citadas pela literatura.

Começando por FFP, a fabricação pode ser definida como a criação de dados ou de resultados. A falsificação é a manipulação de material de pesquisa, equipamentos ou processos, também mudanças ou omissão de dados ou resultados. O plágio é a apropriação indevida de 
ideias, processos, resultados ou palavras utilizadas por outra pessoa (ANDRADE, 2011; BOSSI, 2010; OFFICE OF RESEARCH INTEGRITY, 2008; STENECK, 2002).

$\mathrm{O}$ autoplágio também tem sido apontado como PQ, por ser compreendido como a publicação de um mesmo trabalho (na íntegra ou partes) por um mesmo autor, de forma idêntica ou com pequenas alterações, em mais de um periódico, sem a devida referência ao trabalho já publicado previamente (ANDRADE, 2011; CNPq, 2011; BENOS et al., 2005; CARNEIRO; CANGUSSÚ; FERNANDES, 2007).

Outras ações também têm sido citadas pela literatura como PQs. A fragmentação de dados é a divisão de uma pesquisa em partes menores que se tornam artigos distribuídos em vários periódicos, com a intenção apenas de elevar a quantidade de publicações. A estratégia ganhou outras denominações que direta ou indiretamente, denotam as características que possuem, tais como: produção-salame, ciência-salame, estilo-salame ou fatias-de-salame (ANDRADE, 2011; BENNETT; TAYLOR, 2003; BOSSI, 2010; CASTIEL; SANZ-VALERO, 2007; KUMAR, 2008; MABE; AMIN, 2002; MOJON-AZZI; MOJON, 2004; RIGHETTI, 2010). Assim, se todos os resultados podem ser apresentados de maneira coesiva, dividir em vários estudos, com o intuito único de elevar o número de publicações, não é considerado ético (CNPq, 2012a).

Problema com autoria também é categorizado como uma prática questionável. $\mathrm{O}$ clube da coautoria consiste em um mesmo grupo de pesquisadores que compartilham a autoria em diversos artigos, utilizando critérios duvidosos para atribuir participação. Algumas participações são concedidas em função da utilização de espaços ou equipamentos para coletas de dados, de modo que os líderes do laboratório são "premiados" com o seu nome no artigo. Outras vezes, as "colaborações" podem ocorrer por meras trocas de assinaturas, favores, escambo autoral ou por apenas pertencerem ao grupo do autor principal, entre outros. Também pode acontecer a autoria fantasma, ou seja, quando um indivíduo produz o artigo e seu nome não é incluído na autoria, mas de um pesquisador renomado com o intuito de dar visibilidade para o trabalho (ANDRADE, 2011; BENNETT; TAYLOR, 2003; BOSSI, 2010; CASTIEL; SANZ-VALERO, 2007; BUSSATOFILHO, 2002; GRIEGER, 2005; MONTEIRO et al, 2004; MONTENEGRO, 1999; STENECK, 2006). 
Chama-se a atenção para o fato de que alguns pesquisadores estão na autoria sem ter, efetivamente, participado da elaboração do trabalho. Todavia, a supervisão, obtenção de financiamento e empréstimo de equipamentos não justifica a entrada do indivíduo na autoria e apenas aqueles que contribuíram significativamente merecem entrar como autores do trabalho. Logo, os autores devem ser capazes de deixar claro qual foi a real contribuição de cada autor, quando exigida (CNPq, 2012a; BENNETT; TAYLOR, 2003; INTERNATIONAL COMMITTEE OF MEDICAL JOURNAL EDITORS, 2012?; MONTEIRO et al, 2004).

Existem outras PQs que podem estar relacionadas aos indicadores bibliométricos baseados em citação. $\mathrm{O}$ fator de impacto (FI) e índice $\mathrm{h}$ são amplamente usados para: (a) avaliação da produção de conhecimento no campo científico nacional e internacional (ARAÚJO; SARDINHA, 2011; MUGNAINI, 2006; STREHL, 2005; VOLPATO, 2008); (b) classificação e ranqueamento de pesquisadores em editais de captação de recursos (GRIEGER, 2005; SZKLO, 2008); ou (c) avaliação de promoções acadêmicas, como se fosse possível correlacionar, necessariamente, os números aferidos por esses indicadores bibliométricos em termos de equivalência de qualidade dos artigos publicados (AGRAWAL, 2005; DEMPSEY, 2009).

Obviamente, isso traz outras decorrências, como por exemplo: com a finalidade de aumentar as possibilidades de aceitação de seus artigos, os pesquisadores podem citar autores famosos, com alto índice de citação, ou convidam coautores experientes para garantir a publicação e elevar as chances do estudo ser citado, desconsiderando assim a originalidade (AGRAWAL, 2005; ANDRADE, 2011; KEMPERS, 2002; BENNETT; TAYLOR, 2003; FEESER; SIMON, 2008; LOVISOLO, 2007; MONTEIRO et al., 2004).

Pelo lado dos editores de periódicos, supostamente, pode acontecer a prática chamada de "máfia da citação" (RIGHETTI, 2010, p.A19) e de "corrupção dos FI dos periódicos" (AGRAWAL, 2005, p.157). É possível que alguns editores, para manter o prestígio da revista, dêem preferência para artigos que atraiam citações (KNOBEL, 2003). Outra artimanha é a sugestão ou indução para os autores que submetem trabalhos citarem artigos do próprio periódico visando elevar o FI ou índice $\mathrm{h}$ da revista (AGRAWAL, 2005; KIELING; GOLÇALVEZ, 2007; SEGLEN, 1997; ROIG, 2003). 
A rede complexa de intenções, disputas e lutas do campo científico parece violar a ideia da ciência neutra, sempre presente no discurso público de editores e pesquisadores. Com recursos legítimos (elaboração genuína de um trabalho) ou artificiais (uso das PQs), é construída a figura da "autoridade científica", que significa o alto grau de prestígio social do pesquisador, este que realmente pode estar no efetivo controle do avanço do conhecimento (MERTON, 1968). Logo, as escolhas que supostamente parecem científicas, podem ser escolhas por estratégias sociais que melhor possibilitam a obtenção de reconhecimento dos pares-concorrentes (por exemplo com volume de publicações e índices de citação), sendo este status adquirido o real lucro científico (BOURDIEU, 1976; 2004).

Embora o propósito da produção de conhecimento, supostamente, seja voltado para o avanço de uma determinada área, outras motivações podem estar sendo colocadas como prioridades por afetar diretamente a carreira do pesquisador. Uma delas é a busca pelo prestígio científico.

\section{As práticas questionáveis e o prestígio científico: alguns aponta- mentos}

No campo científico, por ser considerado um espaço de relações sociais, as consequências que uma denúncia ocasiona para a carreira de um pesquisador ou equipe de pesquisa pode fazer com que se estabeleçam normas implícitas de conduta de grupo que afetam a decisão de denunciar ou não uma prática questionável, julgamento este que pode ser dependente da posição hierárquica ocupada. Um dos aspectos que determinam posições na estrutura social é o monopólio da competência científica, ou seja, é a condição legitimada de falar e agir, com autoridade e de modo autorizado, socialmente concedido a uma pessoa e que geralmente ocupa o topo da hierarquia (BOURDIEU, 1976).

$\mathrm{Na}$ relação entre membros de um grupo, indivíduos que detém o monopólio da competência científica podem fazer o uso de sua autoridade para impedir que sejam denunciadas suas próprias PQs, quando ocorrem. Em 2010 foi realizado um estudo com pesquisadores financiados pelo National Institutes of Health (Estados Unidos) para saber como eles lidavam com situações de irregularidades cometidas por colegas de laboratório. Nos resultados ficou claro o peso da autoridade científica de um indivíduo, pois quando o suspeito ocupava cargo su- 
perior no local de pesquisa, as chances de alguém impedir uma PQ eram mínimas. Por outro lado, os resultados apontaram que a probabilidade do pesquisador impedir o comportamento inadequado de outro colega foi maior quando o infrator era estudante de graduação, assistente de pesquisa ou pós-doutor (que fica por um curto período no laboratório) (KOOCHER; KEITH-SPIEGEL, 2010).

Em outras palavras, são maiores as chances de contestar ações duvidosas adotadas pelos novatos no percurso da produção científica, por não possuírem competência científica significativa. Com isso, a maneira como a capacidade científica de um indivíduo será julgada, depende da posição que ocupa na estrutura social hierárquica (BOURDIEU, 1976).

O uso da autoridade científica no emprego de PQs pode ser visto quando uma pessoa faz o uso de sua posição superior para exigir que seu nome seja incluído na autoria de um trabalho realizado por outros, sem ter realizado nenhuma contribuição. Por exemplo, quando um pesquisador é forçado a incluir o nome de outro apenas porque ele possui grande prestígio dentro do grupo ou instituição, tem sido chamado de autoria pressionada (KUMAR, 2008). A coerção de um indivíduo com alto grau de autoridade científica também ocorre quando mentores pressionam seus estudantes para incluir seu nome na autoria, mesmo que não tenha ocorrido nenhuma contribuição por parte do orientador, sendo uma PQ chamada de parasitismo na publicação (KWOK, 2005; KUMAR, 2008). Este tipo de PQ no qual a autoridade científica é empregada para incluir o nome na autoria com base na intimidação também é conhecida como autoria de coerção (CLAXTON, 2005; FEESER; SIMON, 2008).

A autoria de coerção não deveria acontecer ou, até mesmo, não ser comum no meio científico. Todavia, em um estudo realizado no Brasil com 46 estudantes de doutorado em EF, a ação do orientando em incluir o orientador na autoria de um artigo, sem nenhuma contribuição do orientador, foi mencionada como prática não tão rara por $23,9 \%$ dos participantes e $60,9 \%$ apontaram como prática rotineira na área da EF (KUBO, 2012).

Uma das motivações que podem levar o uso das PQs é a cobrança por altos índices de produtividade em pesquisa que, associado à citação, é tido como um indicador de prestígio científico (BOURDIEU, 2004; 2011). Um estudo realizado em 2011 com 85 pesquisadores brasileiros da área de Contabilidade mostrou que eles consideravam a 
exigência de publicação como o aspecto de maior influência na tomada de decisão do sujeito sobre o uso de PQs. Além desses, foram entrevistados oito pesquisadores experientes da área. Eles apontaram como o fator mais importante que pode levar ao comportamento inadequado o princípio do publish or perish, que norteia o sistema de avaliação da pós-graduação stricto-sensu empregada pela Coordenação de Aperfeiçoamento de Pessoal de Nível Superior (CAPES) (ANDRADE, 2011).

Os estudos mencionados apenas representam o cenário de concorrência que é o contexto científico e que pode levar alguns pesquisadores a fazer o uso de todos os meios possíveis para melhorar seus resultados, visando alcançar reconhecimento (PRACONTAL, 2004). No geral, o cenário científico pode ser compreendido como um campo de lutas e a ocupação de posições de prestígio dependem de altos índices de produtividade em pesquisa. Com isso, a ávida busca por publicações passou a ser o principal instrumento para medir o sucesso individual no meio acadêmico e científico brasileiro (TRAGTENBERG, 2002).

No Brasil, o currículo Lattes dos pesquisadores, prioritariamente sustentado pelo volume de publicações em periódicos científicos de alto impacto, passou a ter um valor distintivo no campo científico. O valor diferencial, distintivo, compreende uma espécie particular de capital social que baseia-se na construção de um nome, fundamentado no conhecimento e reconhecimento que lhe garante prestígio e, por sua vez, autoridade no campo científico. Por exemplo, a lógica da distinção se manifesta na posição autoral ocupada, que supostamente depende do valor distintivo de cada nome e pode prevalecer sobre a real contribuição de cada autor para a construção do trabalho (BOURDIEU, 1976).

A disputa por prestígio e todos os lucros decorrentes, financeiro e/ou simbólico, pode levar o pesquisador a usar PQs para inflacionar artificialmente seu currículo, aumentando assim seu valor distintivo. $\mathrm{O}$ valor distintivo de um pesquisador melhora ou piora sua reputação junto aos pares e, em decorrência, torna-o mais ou menos atrativo para estudantes de pós-graduação, bem como aumenta ou diminui chances de obter fundos para sua pesquisa (BOURDIEU, 1976; REIF, 1961).

Hodiernamente, não é possível afirmar que ações irresponsáveis são cometidas, pois a literatura brasileira sobre o tema ainda é incipiente, ainda mais na área da EF. Uma pesquisa realizada em 2011 iden- 
tificou que, entre 2008 e 2010, não foi publicado nenhum artigo na área que abordou a conduta inadequada como temática de estudo. Ao ampliarem a busca para o período entre 2000 e 2010 e para publicações brasileiras de outras áreas, apenas 11 artigos foram encontrados, sendo que nenhum era da EF (KUBO et al, 2011). Porém, um estudo publicado em 2012 adotou a PQ como objeto de pesquisa ao investigar as estratégias que doutorandos dos PPGs em EF tiveram contato ou presenciaram para disseminar o conhecimento científico. Apesar dos resultados não serem generalizados, ao menos mostra que as PQs estão presentes no contexto da pesquisa na EF (KUBO, 2012).

A PQ não é um tema específico da área, mas outras pesquisas que tratam da EF como área de produção e disseminação do conhecimento e, portanto, que está em contato com todo o jogo de interesses que é o campo científico, têm ao menos mencionado as PQs. Assim, estudos brasileiros com foco principal no sistema de avaliação da produtividade em pesquisa têm feito algum tipo de referência às $P Q$ s como uma das estratégias para obter vantagem nas regras do jogo científico (LOVISOLO, 2007; RODRIGUES, 2007; RODRIGUES; REZENDE, 2010; MARCHLEWSKI; MAIA DA SILVA; SORIANO; 2011; VITOR-COSTA; MAIA DA SILVA; SORIANO, 2012). As PQs também foram citadas como um dos motivos que levaram pareceristas a rejeitar artigos submetidos a um importante periódico da área (JOB, MATTOS, ANDRADE, 2009). Também, dois estudos sobre ética em pesquisa na EF abordaram as PQs como má conduta, juntamente com as irregularidades em pesquisa envolvendo seres humanos (ROCHA; BENEDETTI, 2009; SANTANA, 2010).

É de se indagar a razão da falta de publicações na EF que abordam as PQs como temática central de investigação. Assim, a comunidade científica da EF precisa, além de ampliar os estudos sobre as PQs, estar atenta às motivações de porquê o tema ainda não desperta interesse como objeto de pesquisa, uma vez que a $\mathrm{EF}$, como área de conhecimento, está diretamente envolvida com produção científica.

\section{Considerações}

A exigência adotada no Brasil voltada para a produtividade em pesquisa, aliada ao emprego de indicadores bibliométricos baseados em citação para avaliação da produção de conhecimento, são fatores 
que contribuem para o uso de comportamento inadequado tanto dos que a produzem, quanto dos que a avaliam.

As discussões sobre conduta íntegra na pesquisa no país ainda estão em um período gestacional. A criação da Comissão de Integridade na Atividade Científica pelo CNPq é um início promissor e medidas de longo prazo devem ser efetivadas, como a realização de cursos, eventos e disciplinas sobre o tema. Reconhecemos que os cursos de pós-graduação stricto-sensu no país, como principal local de produção científica, deveriam oferecer meios de formação visando a prevenção de ações consideradas questionáveis e a disseminação de uma cultura de boas práticas em pesquisa.

Mesmo com a necessidade em discutir a temática, parece que o debate ainda é incipiente na área da EF. Ainda assim, reconhecemos que a limitação da pesquisa foi restringir a coleta de informações apenas à publicação científica, sendo necessária uma busca mais aprofundada, incluindo, além da literatura publicada, projetos de pesquisa e até mesmo o currículo dos programas de pós-graduação em EF, para compreender melhor o estado da discussão sobre o tema na área. Para estudos futuros, sugerimos que as PQs sejam adotadas como objeto principal de investigação em ambientes destinados à produção de conhecimento científico, como programas de pós-graduação stricto-sensu, bem como em grupos de pesquisa, envolvendo assim os pesquisadores-líderes, alunos de graduação, pós-graduação e pesquisadores-colaboradores. Só assim será possível realizar pesquisas mais detalhadas que ampliem a compreensão do problema, para que a comunidade científica da EF possa elaborar alternativas que estimulem o comportamento responsável de pesquisadores da área.

\title{
SOME REFLECTIONS ON THE RESEARCH ACTIVITY IN PHYSICAL EDUCATION
}

\begin{abstract}
The objective was to present some reflections about what has been discussed by the scientific community in general, and in the field of Physical Education in special, on the theme of questionable practices used in scientific research. Among the numerous strategies to take advantage of the system that evaluates research productivity, the questionable practices rarely appear as the a main objective of research in the field of Physical Education. We conducted a bibliographical research with a qualitative approach, employing content analysis in the selected documents. We believe that more studies about the theme are necessary with the objective of
\end{abstract}


creating effective strategies to prevent questionable practices in research.

Keywords: Scientific Misconduct. Scientific Publication Indicators. Physical Education and Training.

\section{ALGUNAS REFLEXIONES SOBRE EL EJERCICIO DE LA INVESTIGA- CIÓN EN EDUCACIÓN FÍSICA}

\section{Resumen}

El objetivo fue reflexionar sobre lo que ha sido discutido por la comunidad científica en general y el área de Educación Física, sobre las prácticas cuestionables empleados en la realización de investigaciones científicas. Entre las numerosas estrategias para tomar ventaja em el sistema que evalúa la productividad de la investigación, las prácticas cuestionables aparecen con poca frecuencia como el objeto principal de la investigación en el área de Educación Física. El estudio se caracteriza por un enfoque cualitativo, como la literatura. El análisis de contenido se utilizó para analizar los artículos y libros científicos seleccionados. Consideramos que es necesario ampliar los estudios sobre el tema, para encontrar formas efectivas de prevenir prácticas cuestionables en la investigación científica.

Palabras clave: Mala Conducta Científica. Indicadores de Producción Científica. Educación y Entrenamiento Físico.

\section{Referências}

ANDERSON, M. S. Scientific integrity: maintaining the legitimacy of the research enterprise. In: INTERNATIONAL BARCELONA CONFERENCE ON HIGHER EDUCATION: ETHICS AND RELEVANCE OF SCIENTIFIC KNOWLEDGE: WHAT KNOWLEDGE FOR WHAT SOCIETY?, 4., 2008, Barcelona. Proceedings... Barcelona: GUNI. 2008.

ANDRADE, J.X. Má conduta na pesquisa em ciências contábeis. 2011. 115 f. Tese (Doutorado em Ciências Contábeis)-Faculdade de Economia, Administração e Contabilidade, Departamento de Contabilidade e Atuária, Universidade de São Paulo, São Paulo, 2011.

ANGELL M. Publish or perish: a proposal. Annals of Internal Medicine, Philadelphia, v. 104, n. 2, p. 261-262, fev., 1986.

AGRAWAL, A. A. Corruption of journal impact factors. Trends in Ecology \& Evolution, v. 20, n. 4, p.157, 2005.

ARAÚJO, C.G.S; SARDINHA, A. Índice-H dos artigos citantes: uma contribuição para a avaliação da produção científica de pesquisadores 
experientes. Revista Brasileira de Medicina do Esporte, São Paulo, v. 17, n. 5, p. 358-362, set./out., 2011.

AZEVÊDO, E.S. Honestidade científica: outro desafio ao controle social da ciência. Gazeta Médica da Bahia, Salvador, v. 76, n. 1, p. 3541, 2006.

BARDIN, L. Análise de conteúdo. Lisboa: Edições 70, 1977.

BAUER, M. W.; GASKELL, G.; ALLUN, N. C. Qualidade, quantidade e interesses do conhecimento. Evitando confusões. In: BAUER, M. W.; GASKELL, G. Pesquisa qualitativa com texto, imagem e som: um manual prático. Petrópolis: Vozes, 2002. p.17-36.

BENNETT, D. M; TAYLOR, D. M. Unethical practices in authorship of scientific papers. Emergency Medicine, v.15, n. 3, p. 263-70, jun., 2003.

BENOS, D. J. et al. Ethics and scientific publication. Advances in Physiology Education, Bethesda, v. 29, n. 2, p. 59-74, jun., 2005.

BOSSI, E. Scientific integrity, misconduct in science. Swiss Medical Weekly, v. 140, n. 13-14, p. 183-186, 2010.

BOURDIEU, P. Homo academicus. Florianópolis: UFSC, 2011.

. Le champ scientifique. Actes de La Recherche en Sciences Sociales, v. 2, n. 2/3, p. 88-104, 1976.

. Os usos sociais da ciência. São Paulo: Unesp, 2004.

BRASIL. Ministério da Ciência e Tecnologia. Número de artigos brasileiros, da América Latina e do mundo publicados em periódicos científicos indexados pela Thomson/ISI e Scopus, 1981-2011. Brasília: Ministério da Ciência e Tecnologia, 2012. Disponível em: $<$ http://www.mct.gov.br/upd_blob/0224/224830.pdf $>$. Acesso em 18 set. 2012.

BUSSATO-FILHO, G. A importância do uso de critérios objetivos para autoria em artigos científicos. Revista de Psiquiatria Clínica, São Paulo, v. 29, n. 1, p.28-32, 2002. 
CARNEIRO, M. A. A.; CANGUSSÚ, S. D.; FERNANDES, G. W. Ethical abuses in the authorship of scientific papers. Revista Brasileira de Entomologia, São Paulo, v. 51, n. 1, p. 1-5, jan./mar., 2007.

CARVALHO, N.S. Ética no trabalho científico. Arquivos de Gastroenterologia, São Paulo, v. 46, n. 3, p.164-166, jul./set., 2009.

CASTIEL, L.D.; SANZ-VALERO, J. Entre fetichismo e sobrevivência: o artigo científico é uma mercadoria acadêmica? Cadernos de Saúde Pública, Rio de Janeiro, v. 23, n. 12, p.3041-3050, dez., 2007.

CASTRO, F. FAPESP lança Código de boas práticas científicas. Agência FAPESP, 28 set. 2011. Disponível em: $<$ http://agencia.fapesp.br/14551>. Acesso em 30 set. 2011.

CLAXTON, L. D. Scientific authorship. Part 2. History, recurring issues, practices, and guidelines. Mutation Research, Amsterdam, v.589, n.1, p.31-45, jan., 2005.

CONSELHO NACIONAL DE DESENVOLVIMENTO CIENTÍFICO E TECNOLÓGICO (CNPq). Diretrizes. Brasília: [s. n.], 2012a. Disponível em: <http://www.cnpq.br/web/guest/diretrizes $>$. Acesso em: 11 mai. 2012.

CONSELHO NACIONAL DE DESENVOLVIMENTO CIENTÍFICO E TECNOLÓGICO (CNPq). Ética e integridade na prática científica. Relatório da Comissão de Integridade de Pesquisa do CNPq. Bra-

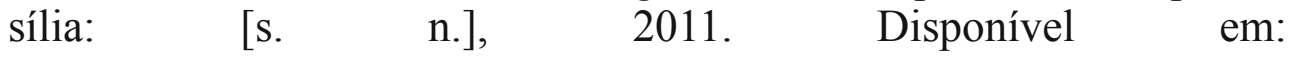
$<$ http://www.cnpq.br/documents/10157/a8927840-2b8f-43b9-89625a2ccfa74dda $>$. Acesso em 30 out. 2011.

CONSELHO NACIONAL DE DESENVOLVIMENTO CIENTÍFICO E TECNOLÓGICO (CNPq). Resolução Normativa $\mathbf{N}^{\circ} 0006 / 2012$. Brasília: $\quad$ s. $\quad$ n.], 2012b. Disponível em: $<$ http://www.cnpq.br/web/guest/view/-/journal_content/56_INSTANCE_0oED/10157/106200>. Acesso em 11 maio 2012.

COMMITTEE ON SCIENCE, ENGINEERING, AND PUBLIC POLICY. On being a scientist: a guide to responsible conduct in research. 3. ed. Washington: The National Academies Press, 2009. 
DE VRIES, R.; ANDERSON, M.S.; MARTINSON, B.C. Normal misbehavior: scientists talk about the ethics of research. Journal of Empirical Research on Human Research Ethics, v.1, n. 1, p.43-50, mar., 2006.

DEMPSEY, J.A. Impact factor and its role in academic promotion: a statement adopted by the International Respiratory Journal Editors Roundtable. Journal of Applied Physiology, v.107, n.4, p.1005, 2009.

FANELLI, D. How many scientists fabricate and falsify research? A systematic review and meta-analysis of survey data. PloS One, San Francisco, v. 4, n. 5, p. 5738, maio, 2009.

FARTHING, M.J.G. 'Publish, and be damned...' the road to research misconduct. Journal of the Royal College of Physicians of Edinburg, v. 34, n. 4, p.301-304, 2004.

FEESER, V.R; SIMON, J.R. The ethical assignment of authorship in scientific publications: issues and guidelines. Academic Emergency Medicine, Philadelphia, v. 15, n. 10, p. 963-969, out., 2008.

GRIEGER, M.C.A. Authorship: an ethical dilemma of science. São Paulo Medical Journal, São Paulo, v. 123, n. 5, p. 242-246, set., 2005.

. Escritores-fantasma e comércio de trabalhos científicos na internet: a ciência em risco. Revista da Associação Médica Brasileira, São Paulo, v. 53, n. 3, p.27-28, maio/jun., 2007.

INTERNATIONAL COMMITTEE OF MEDICAL JOURNAL EDITORS (ICMJE). Uniform requirements for manuscripts submitted to biomedical journals. Disponível em: $<$ http://www.icmje.org/ethical_1author.html>. Acesso em 05 abr. 2012.

JOB, I.; MATTOS, A.M.; TRINDADE, A. Processo de revisão pelos pares: por que são rejeitados os manuscritos submetidos a um periódico científico? Movimento, Porto Alegre, v. 5, n. 3, p. 35-55, jul./set., 2009. 
JOHN, L. K.; LOEWENSTEIN, G.; PRELEC, D. Measuring the prevalence of questionable research practices with incentives for truth telling. Psychological Science, v. 23, n. 5, p. 524-532, 2012.

KEMPERS, R.D. Ethical issues in biomedical publications. Fertility and Sterility, Nova York, v. 77, n. 5, p.883-888, maio, 2002.

KIELING, C.; GONÇALVES, R.R.F. Assessing the quality of a scientific journal: the case of Revista Brasileira de Psiquiatria. Revista Brasileira de Psiquiatria, São Paulo, v. 29, n. 2, p. 177-181, jun., 2007.

KNOBEL, M. Fraudes sacodem a comunidade científica. Ciência e Cultura, São Paulo, v. 55, n. 3, p. 17-18, jul./set., 2003.

KOOCHER, G. P.; KEITH-SPIEGEL, P. Peers nip misconduct in the bud. Nature, v. 466, n. 7305, p.438-440, jul., 2010.

KUBO, K. C. M. L. Pós-graduação em educação física e produção de conhecimento: estratégias para disseminação científica. 2012. 136 f. Dissertação (Mestrado em Educação Física)-Programa de PósGraduação em Educação Física Associado UEL/UEM, Departamento de Educação Física, Centro de Educação Física e Esporte, Universidade Estadual de Londrina, Londrina, 2012.

KUBO, K. C. M. L. et al. Má conduta e a produção de conhecimento na educação física. Motriz, Rio Claro, v. 17, n. 1, p. S89, jan./mar., 2011. Suplemento 1.

KUMAR, M.N. A review of the types of scientific misconduct in biomedical research. Journal of Academic Ethics, v. 6, n. 3, p. 211-118, 2008.

KWOK, L. S. The White Bull effect: abusive coauthorship and publication parasitism. Journal of Medical Ethics, v.31, n.9, p.554-556, set., 2005.

LOVISOLO, H.R. "Levantando o sarrafo ou dando tiro no pé": critérios de avaliação e Qualis das pós-graduações em educação física. Revista Brasileira de Ciências do Esporte, Porto Alegre, v. 29, n. 1, p. 23-33, 2007. 
LUNA, S. V. Planejamento de pesquisa: uma introdução. São Paulo: EDUC, 1999.

MABE, M.A; AMIN, M. Dr. Jekyll and Dr. Hyde: author-reader asymmetries in scholarly publishing. Aslib Proceedings, v. 54, n. 3, p. 149-157, 2002.

MACRINA, F.L. Scientific integrity: text and cases in responsible conduct of research. 3. ed. Washington: ASM Press, 2005.

MANCINI, M. C.; SAMPAIO, R. F. Quando o objeto de estudo é a literatura: estudos de revisão. Revista Brasileira de Fisioterapia, São Carlos, v. 10, n. 4, out./dez., 2006 . Não paginado.

MARCHLEWSKI, C.; MAIA DA SILVA, P.; SORIANO, J. B. A influência do sistema de avaliação Qualis na produção de conhecimento científico: algumas reflexões sobre a Educação Física. Motriz, Rio Claro, v. 17, n. 1, p.104-116, jan./mar., 2011.

MARCONI, M. A.; LAKATOS, E. M. Fundamentos de metodologia científica. 5. ed. São Paulo: Atlas, 2003.

MARTINSON, B.C; ANDERSON, M.S.; DE VRIES, R. Scientists behaving badly. Nature, v. 435, n. 704, p. 737-738, jun., 2005.

MERTON, R.K. The Matthew Effect in science. Science, v. 158, n. 3810, p. 56-63, 1968.

MINAYO, M. C. S. Pesquisa social: teoria, método e criatividade. Petrópolis: Vozes, 1994.

MOJON-AZZI, S.M.; MOJON, D.S. Scientific misconduct: from salami slicing to data fabrication. Ophthalmologica, Basel, v. 218, n. 1, p. 1-3, jan./fev., 2004.

MONTEIRO, R. et al. Critérios de autoria em trabalhos científicos: um assunto polêmico e delicado. Revista Brasileira de Cirurgia Cardiovascular, São José do Rio Preto, v. 19, n. 4, p.III-VIII, nov./dez., 2004. 
MONTENEGRO, M. R. Autoria e co-autoria: justificativa e desvios. Jornal de Pneumologia, Brasília, v. 25, n. 3, p. 159-162, maio/jun., 1999.

MOREIRA, W. Revisão de literatura e desenvolvimento científico: conceitos e estratégias para confecção. Janus: Revista de Pesquisa Científica, Lorena, v. 1, n. 1, p.19-30, 2004.

MUGNAINI, R. Caminhos para adequação da avaliação da produção científica brasileira: impacto nacional versus internacional. 2006. 253 f. Tese (Doutorado em Ciência da Informação)-Escola de Comunicação e Artes, Universidade de São Paulo, São Paulo, 2006.

OFFICE OF RESEARCH INTEGRITY (ORI). 2008 annual report. Washington, DC: [s. n.], 2008.

PANEL ON SCIENTIFIC RESPONSIBILITY AND THE CONDUCT OF RESEARCH. Responsible Science: ensuring the integrity of the research process. Washington: National Academy Press. 1992.

PETROniaU, A. Autoria de um trabalho científico. Revista da Associação Médica Brasileira, São Paulo, v. 48, n. 1, p. 60-75, jan./mar., 2002.

. Critérios para autoria e avaliação de uma publicação científica. Revista de Psiquiatria Clínica, São Paulo, v.37, n.1, p.1-5, jan., 2010.

PRACONTAL, M. A impostura científica em dez lições. Campinas: Unesp, 2004.

REIF, F. The competitive world of the pure scientist. Science, v. 134, n. 3494, p. 1957-1962, 1961.

RICHARDSON, R. J. Pesquisa social: métodos e técnicas. São Paulo: Atlas, 1985.

RIGHETTI, S. Artimanhas inflam produção científica. Folha de São Paulo, 11 jul. 2010. 
ROCHA, J. C. S.; BENEDETTI, T. R. B. Ética na pesquisa em Educação Física. Revista Brasileira de Cineantropometria \& Desempenho Humano, Florianópolis, v. 11, n. 3, p. 358-362, 2009.

RODRIGUES, L. O. C. Publicar mais, ou melhor? O tamanduá olímpico. Revista Brasileira de Ciências do Esporte, Porto Alegre, v. 29, n. 1, p. 35-48, 2007.

RODRIGUES, L. O. C.; REZENDE, N. A. O tamanduá olímpico a caminho da obesidade científica. Revista Médica de Minas Gerais, v. 20 , n. 3 , p. $375-379,2010$.

RODRIGUES, A. V. F.; CRESPO, I. M.; MIRANDA, C. L. Ética em pesquisa e publicações científicas. Em Questão, Porto Alegre, v. 12, n. 1, p. 33-50, 2006.

ROIG, M. Avoiding plagiarism, self-plagiarism, and other questionable writing practices: a guide to ethical writing. 2003. Disponível em: <http://www.cse.msu.edu/ alexliu/plagiarism.pdf $>$. Acesso em: 17 maio 2011.

SANTANA, C. C. O tema da integridade científica nas pós-graduações em saúde no Brasil. Revista Bioética, v. 18, n. 3, p. 637-644, 2010.

SÁ-SILVA, J. R.; ALMEIDA, C. D.; GUINDANI, J. F. Pesquisa documental: pistas teóricas e metodológicas. Revista Brasileira de História \& Ciências Sociais, v. 1, n. 1, p. 1-14, 2009.

SCOPUS. Country Ranking - 2010. SCImago. 2010. Disponível em: $<$ http://www.scimagojr.com/countryrank.php?area $=0$ \&category $=0 \&$ region $=$ all\&year $=2010 \&$ order $=i t \& \min =0 \&$ min_type $=i t$.

Acessado em 05 de agosto de 2012>.

SEGLEN, P. Why the impact factor of journals should not be used for evaluating research. British Medical Journal, Londres, v. 314, n. 7079, p. 498-502, fev., 1997.

STENECK, N.H. Assessing the integrity of publicly funded research. In: ORI RESEARCH CONFERENCE ON RESEARCH INTEGRITY, 1., 1997, Washington. Proceedings... Washington-DC: Office of Research Integrity, p.1-16, 2002. 
STENECK, N.H. Fostering integrity in research: definitions, current knowledge, and future directions. Science and Engineering Ethics, Guildford, v. 12, n. 1, p. 53-74, jan., 2006.

STREHL, L. O fator de impacto do ISI e a avaliação da produção científica: aspectos conceituais e metodológicos. Ciências da Informação, Brasília, v. 34, n. 1, p. 19-27, jan./abr., 2005.

SZKLO, M. Impact factor: good reasons for concern. Epidemiology, Cambridge, v. 19, n. 3, p. 369, maio, 2008.

TAVARES-NETO, J. Destaques éticos nos periódicos nacionais das áreas médicas. Revista da Associação Médica Brasileira, São Paulo, v. 55 , n. 4 , p. $400-404,2009$.

TRAGTENBERG, M. A delinquência acadêmica. Revista Espaço Acadêmico, v. 2, n. 14, p. 12-15, 2002.

TURATO, E. R. Métodos qualitativos e quantitativos na área da saúde: definições, diferenças e seus objetos de pesquisa. Revista de Saúde Pública, São Paulo, v. 39, n. 3, p. 507-514, jun., 2005.

VASCONCELOS, S. et al. Discussing plagiarism in Latin American science. Brazilian researchers begin to address an ethical issue. EMBO Reports, Oxford, v. 10, n. 7, p. 677-682, jul., 2009.

VITOR-COSTA, M.; MAIA DA SILVA, P.; SORIANO, J. B. A avaliação da produtividade em pesquisa na Educação Física: reflexões sobre algumas limitações dos indicadores bibliométricos. Revista Brasileira de Educação Física e Esporte, São Paulo, v. 26, n. 4, p. 581-597, out./dez., 2012.

VOLPATO, G.L. Indicadores de qualidade da publicação científica. Tropical Plant Pathology, v. 33, p.S42-S44, ago., 2008. Suplemento.

Recebido em: 31/10/2012

Revisado em: 24/01/2013

Aprovado em: 28/02/2013 


\section{Endereço para correspondência}

jbsoriano1967@gmail.com

Jeane Barcelos Soriano

Universidade Estadual de Londrina,

Centro de Educação Física e Desportos,

Departamento de Fundamentos da Educação Física.

Rodovia Celso Garcia Cid - Pr 445 - Km 380

Jardim Portal de Versalhes 1

86051-990 - Londrina, PR - Brasil - Caixa-postal: 6001 